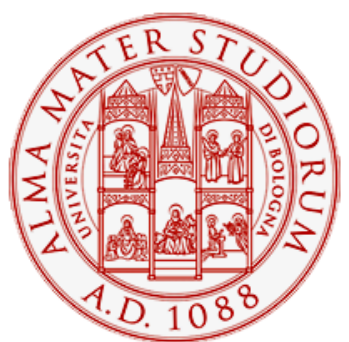

Alma Mater Studiorum - Università di Bologna DEPARTMENT OF ECONOMICS

Short-Run Regional Forecasts:Spatial Models through Varying Cross-Sectional and Temporal Dimensions

Matías Mayor-Fernández Roberto Patuelli

Quaderni - Working Paper DSE $N^{\circ} 835$

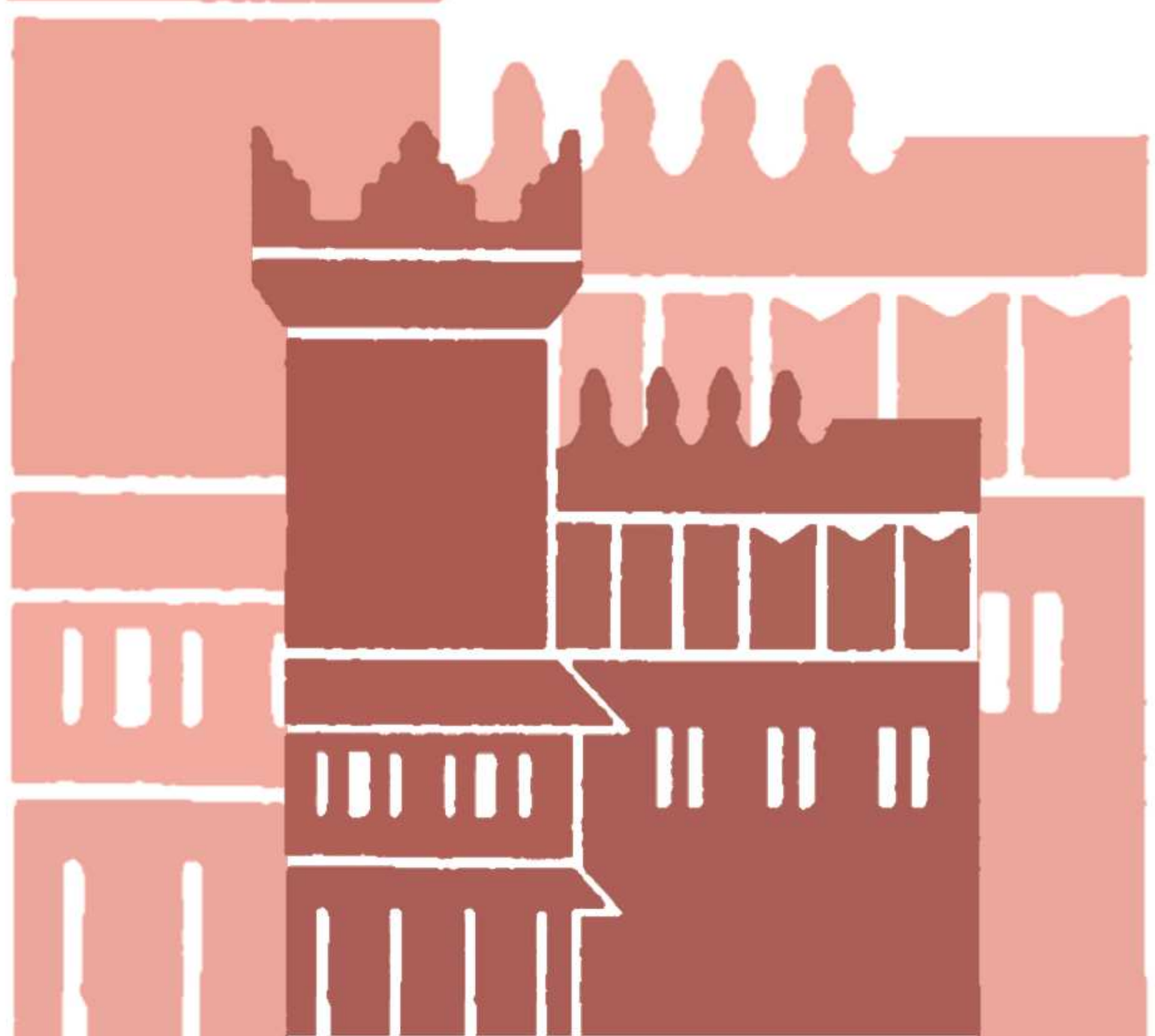




\title{
Short-Run Regional Forecasts: Spatial Models through Varying Cross-Sectional and Temporal Dimensions
}

\author{
Matías Mayor-Fernández ${ }^{1}$ and Roberto Patuelli ${ }^{2}$
}

${ }^{1}$ Department of Applied Economics, University of Oviedo, Spain. Email: mmayorf@uniovi.es;

2 Department of Economics, Faculty of Economics-Rimini, University of Bologna, Italy; The Rimini Centre for Economic Analysis (RCEA), Italy. E-mail: roberto.patuelli@unibo.it.

\begin{abstract}
In any economic analysis, regions or municipalities should not be regarded as isolated spatial units, but rather as highly interrelated small open economies. These spatial interrelations must be considered also when the aim is to forecast economic variables. For example, policy makers need accurate forecasts of the unemployment evolution in order to design short- or long-run local welfare policies. These predictions should then consider the spatial interrelations and dynamics of regional unemployment. In addition, a number of papers have demonstrated the improvement in the reliability of long-run forecasts when spatial dependence is accounted for. We estimate a heterogeneouscoefficients dynamic panel model employing a spatial filter in order to account for spatial heterogeneity and/or spatial autocorrelation in both the levels and the dynamics of unemployment, as well as a spatial vector-autoregressive (SVAR) model. We compare the short-run forecasting performance of these methods, and in particular, we carry out a sensitivity analysis in order to investigate if different number and size of the administrative regions influence their relative forecasting performance. We compute short-run unemployment forecasts in two countries with different administrative territorial divisions and data frequency: Switzerland (26 regions, monthly data for 34 years) and Spain (47 regions, quarterly data for 32 years)
\end{abstract}




\section{Introduction}

Forecasting economic values in administrative units provides very important information for political, institutional and economic agents for their respective planning processes. A crucial stage is the choice of the econometric method to obtain these future values taking into account the diversity and complexity of real economy. Two aspects may be considered when choosing an econometric specification. Firstly, disparities in economic development and welfare within countries (i.e., at the regional level) are often bigger than between countries (Elhorst 1995; Taylor and Bradley 1997; Ertur and Le Gallo 2003; Patuelli 2007; see, for example, the cases of Germany and Spain), and they often show typical geographical/spatial structures. Secondly, with regard to regional unemployment disparities, policy makers need, in order to correctly target their actions and policies, to understand two aspects of such disparities: (a) the determinants of 'equilibrium' unemployment and its variation; and, (b) the region-specific and the cross-regional dynamics of unemployment. On the one hand, the need for an explicit consideration of the existence of spatial interdependence in econometric models, which is consistent with regional science theories asserting the importance of spatial linkages in local economic processes, led to what is nowadays quite a large literature of empirical papers. On the other hand, the temporal perspective of the problem has attracted less attention in spatial models, but should be considered jointly.

The spatial perspective has achieved an increasing relevance within the field of labour market studies. Some recent contributions have taken into account the spatial dimension of regional labour markets and pointed out the high degree of interdependence of local labour markets (Molho 1995; López-Bazo et al. 2002; Overman and Puga 2002). Furthermore, Patacchini and Zenou (2007) analyse the reasons for spatial dependence in local unemployment rates. This spatial autocorrelation is mainly due to the fact that unemployed individuals may seek and find work in different areas, so that spatial interactions result from their mobility. When the data is collected at the administrative level, as it is often the case, spatial autocorrelation is likely to be a relevant issue.

The contribution to forecasting power of the inclusion of dependence across spatial units has been analysed in several papers. For example, Giacomini and Granger (2004, p. 7), on the one hand, stress that 'ignoring spatial autocorrelation, even when it is weak, leads to highly inaccurate forecasts'. On the other hand, Hernández-Murillo and Owyang (2006) find reductions in the out-of-sample mean squared error (MSE) when employment forecasts are obtained using disaggregated data in a space-time autoregressive model without contemporaneous influence from a region's neighbours ${ }^{1}$ incorporating spatial interaction. Different econometric techniques have been proposed in the literature. Using static spatial panel data models, Baltagi and Li (2004, 2006), as well as Longhi and Nijkamp (2007), forecast employment in West German regions, while Fingleton (2009) predicts the average wage rate across all occupations in local administrative units of Great Britain. A dynamic spatial panel data model is used by Khoodilin et al. (2008) conclude that accounting for spatial effects improves forecast performance, and this improvement is more important when the forecasting horizon is longer. Schanne et al. (2010) reach a similar conclusion comparing a univariate spatial GVAR model with univariate time series methods.

\footnotetext{
${ }^{1}$ These authors assume that these effects are expanded through the time dimension.
} 
In this paper, we compare different methods to obtain short-run unemployment forecasts in (small) administrative units, and observe their performance between different countries. Our interest here is to exploit the strong heterogeneity in the size (e.g., in terms of population or area) of NUTS regions at the same level of aggregation across countries to investigate the variation in the performance of different spatial econometric methods. We analyse the forecasting performance of two competing econometric methods: a spatial vector autoregressive (SVAR) model (Beenstock and Felsenstein 2007; Kuethe and Pede 2011) and a dynamic heterogeneous-coefficients panel data model based on an eigenvector-decomposition spatial filtering (SF) procedure (Griffith 2000, 2003). The two models chosen belong to two separate traditions: VAR models represent the mainstream (time-series) forecasting tradition, while the SF-enhanced dynamic panel model attempts to merge the panel data modelling tradition to the spatial statistics one, within a semi-parametric framework.

The remainder of the paper is structured as follows. In Section 2, SVAR models and SF procedures are described, pointing out their advantages and disadvantages. In Section 3, we discuss the spatial configuration of regional unemployment data in Spain and Switzerland, and subsequently present our forecasting experiment strategy. Our forecasting results and related multidimensional measures of accuracy are included in Section 4. Section 5 concludes the paper.

\section{Modelling Spatio-Temporal Data: Spatial VAR Models and Spatial Filtering}

A VAR model (Sims 1980) can be written as a set of symmetric equations in which each (dependent) variable is described by a set of its own lags and the lags of other variables in the system. VAR models are considered as the most popular method to study the linkages among several variables with high flexibility since these types of models are not based on any theoretical structure. Restrictions are imposed to a large extent by statistical tools rather than by prior beliefs supported by uncertain theoretical models. However, this flexibility is only certain in the temporal dimension. ${ }^{2}$ A standard VAR model assumes the no existence of spatial spillovers, for example, a shock in one region only influence the economic behaviour of this administrative area.

There are a few proposals in the literature on how to introduce spatial relationships between neighbours in a VAR framework, thus relaxing this limitation. This is due to the fact that the number of parameters which are needed to collect such neighbouring relations increase quadratically with the number of spatial units. Therefore, some of the existing proposals use spatial contiguity information to limit the number of parameters. In particular, Pan and LeSage (1995) propose to use spatial contiguity information as an alternative prior in a Bayesian VAR model. Following the same idea, Di Giacinto (2003) defines parameter constraints in a structural VAR model based on neighbouring structure, allowing the identification and estimation of the spatial VAR model. A further option is developed by Schanne et al. (2010), based on the Global VAR (GVAR) model proposed by Pesaran et al. (2004), where geographical information is used to include

\footnotetext{
${ }^{2}$ Structural VAR models are introduced to incorporate some necessary restrictions which are not tested by statistical tools.
} 
spatial connections between regions. One of the novelties (or advantages) of the GVAR model consists in the inclusion of a temporal dimension within the spatial dependence process. Some authors consider only contemporaneous spatial processes (Longhi and Nijkamp 2007; Kholodilin et al. 2008), whereas others specify only a temporally lagged type of spatial dependence (Hernández-Murillo and Owyang 2006).

Regarding the approach to including neighbouring linkages between spatial units, the contributions mentioned above use spatial weights matrices. Spatial weights matrices are positive, non-stochastic and their elements show the intensity of interdependence between pairs of spatial units, that is, eventually specify the neighbouring set for each spatial unit. In this paper, we follow the SVAR approach proposed by Beenstock and Felsenstein (2007), where traditional VAR methods and modern spatial panel data techniques are 'mixed'. Beenstock and Felsenstein allow for both contemporaneous and serially lagged spatially correlated variables. This SVAR model is highly nonlinear, because of the contemporaneous spatial autoregressive process. Its proponents restrict the coefficient of the endogenous contemporaneous spatial lag to zero, therefore linearizing the model.

Let us consider a country divided into $N$ regions or municipalities $(i=1, \ldots, n)$ where the values of a set of random variables are observed over time $t=1, \ldots, T$. In general terms, if we consider $p$ temporal lags and $s$ spatial cross-regressive lags, we must manage $n$ equations like the following (one for each region):

$$
y_{i, t}=c_{i}+\sum_{p=1}^{P} \beta_{i, p} y_{i, t-p}+\sum_{s=1}^{S} \delta_{i, s} W_{s} y_{i, t}+\sum_{s=1}^{S} \sum_{p=1}^{P} \gamma_{i, s, p} W_{s} y_{i, t-p}+\varepsilon_{i, t} \text {, }
$$

where $W_{s}$ is the spatial weight matrix. The novelty of this model is the inclusion of the spatial cross-regressive lags. They are obtained by premultiplying each temporally lagged value by the spatial weight matrix (different contiguity orders could ideally be considered). A further relevant advantage of this approach is the possibility of testing for the significance of regional spillovers by means of Granger causality test.

In the estimation stage, it is necessary to bear in mind that each spatial unit has a unique value of the spatial lag variable, and each observation has its own set of neighbouring units. Since $W_{s} y_{i, t}$ and $\varepsilon_{i, t}$ are not independent, Equation (1) cannot be estimated directly. The system is estimated by seemingly unrelated regressions (SUR), because a two-stage estimation procedure is necessary, where the coefficients of the contemporary spatial lags are estimated using the (spatially weighted) predicted values of the dependent variable as instruments. These predicted values are computed using the reduced form of Equation (1). This is the common solution employed to solve the endogeneity issue caused by the spatial lag of the dependent variable in a simple spatial lag model.

If the order of the temporal lags is restricted to one to preserve degrees of freedom, each equation includes a constant, the lagged variable, and the contemporaneous and temporally lagged spatially correlated variables, as follows:

$$
y_{i, t}=c_{i}+\beta_{i, 1} y_{i, t-1}+\gamma_{i, 1,1} W_{1} y_{n, t-1}+\delta_{i, 1} W_{1} y_{n}+\varepsilon_{i, t}
$$


where $W_{1} y_{n}$ collects the value of contemporaneous spatially lagged variables, and $W_{1} y_{n, t-1}$ the temporally and spatially lagged variables. The accuracy of this type of models in terms of forecasting errors has been previously analysed in the aforementioned papers.

The aim of this paper is to compare this method with the one recently proposed by Patuelli et al. (2012), based on a heterogeneous-coefficients dynamic panel data model enhanced by spatial filtering (SF). This latter approach allows us to account for spatial heterogeneity and/or autocorrelation both in the levels and in the regression coefficients, among which the one of the serially lagged term.

Eigenvector-decomposition SF (Griffith 2000, 2003) is a nonparametric solution to the problem of spatial autocorrelation in regression models. The method relies on the computational formula of Moran's I (MI, Moran 1948) - the most commonly employed statistical indicator for spatial autocorrelation - which is given by:

$$
I=\frac{N \sum_{i} \sum_{j} w_{i j}\left(x_{i}-\bar{x}\right)\left(x_{j}-\bar{x}\right)}{\left(\sum_{i} \sum_{j} w_{i j}\right) \sum_{i}\left(x_{i}-\bar{x}\right)^{2}} .
$$

In Equation (3), $x_{i}$ is the value of the variable $X$ in the $i$ th region, and $w_{i j}$ is the $(i, j)$ element of the spatial weights matrix $\mathbf{W}$. After pre- and post-multiplying $\mathbf{W}$ by a projection matrix, we obtain:

$$
\mathbf{C}=\left(\mathbf{I}_{n}-\mathbf{1 1}{ }^{\mathrm{T}} / n\right) \mathbf{W}\left(\mathbf{I}_{n}-\mathbf{1 1}^{\mathrm{T}} / n\right)
$$

where $\mathbf{1}$ is an $n \times 1$ vector of 1 's. Matrix $\mathbf{C}$ can actually be used to obtain, given variable $X$, the numerator of MI (Equation (3)), and its extreme eigenvalues are approximately the extreme values of MI (Griffith 2000). Because of this mathematical relation between $\mathbf{C}$ and MI, the eigenvectors extracted from $\mathbf{C}$ represent all mutually exclusive (orthogonal and independent) spatial patterns implied by the chosen spatial weights matrix $\mathbf{W}$. The eigenvectors $E_{1} \ldots E_{n}$ of $\mathbf{C}$ are extracted in decreasing order of spatial autocorrelation (MI). Therefore, $E_{1}$ has the largest MI achievable, given the choice of W. All subsequent eigenvectors maximize MI while being orthogonal to all previously extracted eigenvectors.

When employed in a regression model framework as additional explanatory variables, such eigenvectors may account, among other things, for unobserved heterogeneity, redundant information, and spatial spillover effects, rendering regression residuals spatially uncorrelated (at least in a cross-sectional framework). A stepwise regression approach may be used to select which eigenvectors are actually significant in a specific modelling exercise. Because the number of eigenvectors increases with the crosssectional dimension, starting with a subset of so-called 'candidate' (or 'dominant') eigenvectors is convenient. This subset is usually defined according to a threshold of 0.25 for the ratio $I\left(E_{k}\right) / \max _{k} I_{k}$ (for details, see Griffith 2003). The linear combination of the set of $k$ eigenvectors resulting from the selection procedure and their estimated regression coefficients is called a 'spatial filter'. 
Additionally, SF may be employed to inspect the spatial heterogeneity of regression coefficients, equivalently to what is done in geographically weighted regression (GWR, Fotheringham et al. 2002). ${ }^{3}$ Patuelli et al. (2012) show that, in a dynamic panel modelling framework, a heterogeneous-coefficients model can be successfully approximated by constructing a spatial filter-representation of the vector of serial autoregressive coefficients, simultaneously allowing for improved inference in unit root testing. This may be done by interacting each candidate eigenvector (repeated $T$ times) with the serially lagged (dependent) variable, thus constructing a set of new variables representing its spatial decomposition in orthogonal components. The regression coefficients associated to these new variables will indicate relevance of spatial patterns in adjustment processes. The same process can be applied to any other explanatory variable (e.g., to seasonal indicator variables).

When spatial filters are simultaneously applied to the serial correlation coefficient and at intercept level, the following model is obtained:

$$
y_{i, t}=c+\beta y_{i, t-1}+\sum_{m=1}^{k} \beta_{m} E_{i, m} y_{i, t-1}+\sum_{m^{\prime}=1}^{k^{\prime}} \beta_{m^{\prime}} E_{i, m^{\prime}}+\varepsilon_{i, t}
$$

where $k$ and $k^{\prime}$ are the number of eigenvectors selected at the lagged term and intercept level, respectively, and $m$ and $m^{\prime}$ are counters for the selected eigenvectors, again for the same two spatial filters. A (standard) intercept $c$ and an average serial correlation coefficient $\beta$ are still estimated. The two spatial filters obtained provide the regional deviations from these aggregate measures.

\section{Data and Forecasting Strategy}

We test the forecasting performance of the two methods presented above on two data sets relating to Spain and Switzerland. As a numerical example, we use official regional unemployment rates at the NUTS-3 level of geographical aggregation, and analyse the temporal evolution of forecasting errors and their spatial distribution.

We choose Spain and Switzerland since two desired characteristics for this comparison exercise are verified. On the one hand, both data sets have satisfactory but different temporal $(T)$ and spatial dimensions $(n)$. On the other hand, the geographical size of the spatial (administrative) units analysed is widely different. The average area of Spanish provinces is about $10,499 \mathrm{~km}^{2}\left(\sigma=4,699.77 \mathrm{~km}^{2}\right)$, whereas the same for the Swiss cantons is $1582 \mathrm{~km}^{2}\left(\sigma=1,822.35 \mathrm{~km}^{2}\right)$. Therefore, the size of the Swiss cantons is lower with a high level of variability.

Unemployment data for Spain are collected through the Spanish Labour Force Survey (Encuesta de Población Activa, EPA). The data consist of quarterly unemployment rates by province (corresponding to the Spanish NUTS-3 level of geographical classification) and cover the period 1977-2006. Most studies about Spanish labour markets assert that

\footnotetext{
${ }^{3}$ Griffith (2008) stresses that the SF-based approach to GWR actually provides superior statistical properties (e.g., with regard to multicollinearity) than the original GWR.
} 
one of its main features is the unemployment persistence from an aggregate viewpoint, but the persistence of differences in unemployment rates across provinces is highlighted as well (Blanchard and Jimeno 1995; Jimeno and Bentolila 1998). In 2006, we can find some provinces with high unemployment rates (above 14 per cent, like Cádiz, Badajoz, Huelva and Cordoba), whereas others (Teruel, Soria, Navarra and Guipuzcua) have rates lower than 6 per cent. These relevant differences are quite similar along the entire period studied.

Switzerland is a non-EU country, and its labour market can be considered to be quite different from the EU average. Although some constraints have been relaxed with regards to employment and migration regulations (Switzerland has recently eliminated immigration quotas, and started participating in the Schengen agreement), the Swiss labour market is still strictly regulated, and migration is controlled through working permits.

Unemployment data for Switzerland are given through the Unemployment Statistics of the Swiss Federal Statistical Office. The data set we employ consists on monthly unemployment rates between 1975 and 2006, collected for the 26 cantons of Switzerland (again, corresponding to the NUTS-3 geographical aggregation level). Unemployment rates in Switzerland are much lower than in Spain. From 1995 to 2010, Switzerland's unemployment rate averaged 3.38 per cent, reaching an historical high of 5.40 per cent in 1997, and a record low of 1.60 per cent in 2000 . The difference between Switzerland's historical high and the unemployment rates in Spain is striking.

The temporal evolution of unemployment rates is also quite different, and Spanish data show a higher level of volatility as it is shown in Figure 1.

(a)

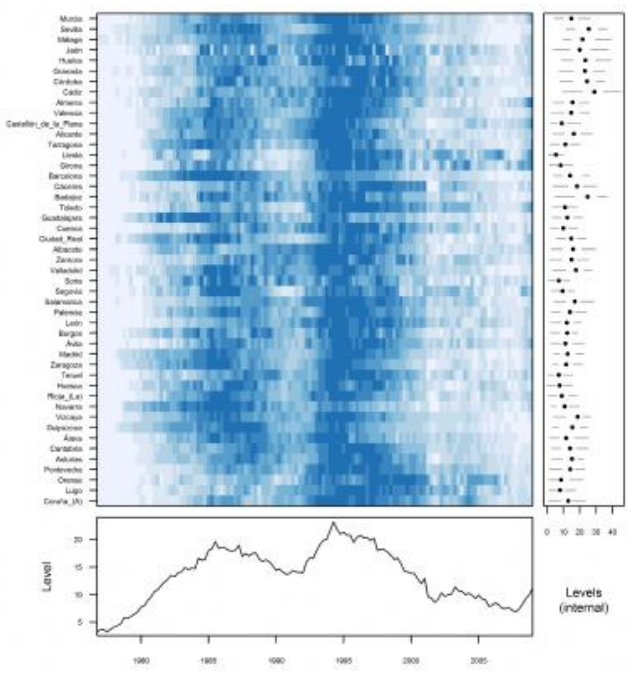

(b)

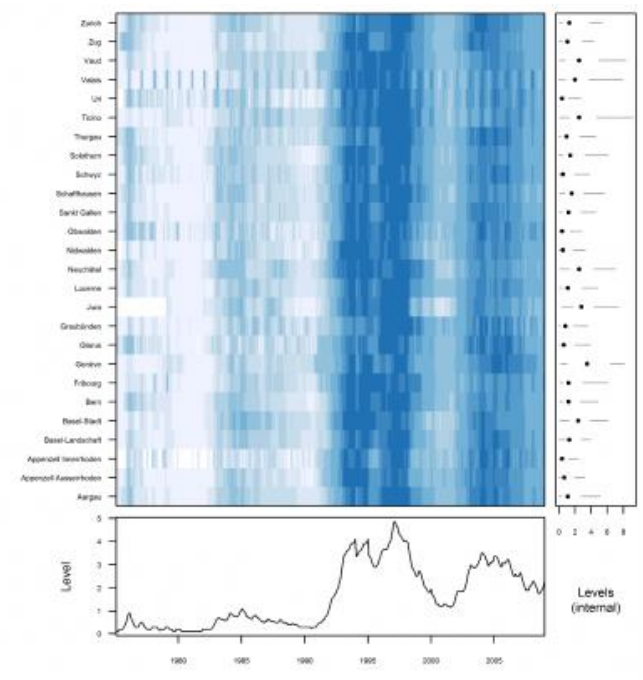

Figure 1 - Panel plots of regional unemployment rates for Spain (a) and Switzerland (b)

The two plots composing Figure 1 cover comparable time periods, and employ, for each single region, a separate colour scale, based on quantiles. The underlying graphs given the evolution of the regional unemployment rate at the median. As it can be seen, most Spanish regions experienced two highs in unemployment, around 1985 and 1995, followed by a marked improvement and, ultimately, by the first signs of unemployment rise coinciding with the 2008 financial crisis. Swiss regions, instead, experienced a 
marked unemployment rate increase between 1990 and 1995, which lasted until about 2000. It is worth noting that, while Swiss regions all follow the aggregate trend (the lighter and darker parts of the plot are homogeneous by row), not all Spanish regions do, suggesting a possible heterogeneity in cyclical sensitivity, which could be reflected in spatial patterning of serial correlation coefficients.

As noted above, spatial interactions between spatial units are highly relevant in a labour market context, and they are often introduced by means of a spatially lagged variable. Although there are different ways to model connectivity between areas, spatial weight matrices used in this paper are based on the contiguity criterion, where a weight of 1 is assigned to the cell $(i, j)$ of $\mathbf{W}$ if the spatial units $i$ and $j$ share a common boundary, and 0 otherwise. The matrices are row-standardized in the case of the SVAR, and globally standardized (Tiefelsdorf et al. 1999) for SF, because of the symmetry requirement for eigenvector extraction.

Before choosing to include spatial structure in our forecasting models, we test for the existence of spatial autocorrelation in our data, using average values per year. ${ }^{4}$ Significant positive spatial autocorrelation is found, which suggests the existence of spillovers across regions.

In order to evaluate the short-run predictive power of the two compared methods, we devise a forecasting strategy based on a rolling window. For each model and data set, estimates are obtained using a fixed window of observations, between $t=1+g$ and $t=T-h+g$, and ex post forecasts of regional unemployment rates are carried out for the nearest subsequent time period, for $g=0, \ldots, h-1$ and $h$ being the number of time periods covered by the forecasting window. The forecasting window moves over two years, therefore providing forecasts over 8 quarters for Spain and 24 months for Switzerland. Given cross-sectional dimensions, the overall number of forecasted values is $(8 * 47=) 376$ for Spain and $(24 * 26=) 624$ for Switzerland.

\section{Results}

The forecasting performance of the SVAR and SF methods is summarized and compared by means of statistical indicators: the mean square error (MSE), the mean absolute error (MAE) and the mean absolute percentage error (MAPE). The MSE and MAE measure deviations in absolute value from the true values, and are computed as follows:

$$
\begin{gathered}
M S E=\frac{1}{n} \sum_{i=1}^{n}\left(\hat{U}_{i}-U_{i}\right)^{2} ; \\
M A E=\frac{1}{n} \sum_{i=1}^{n}\left|\hat{U}_{i}-U_{i}\right| .
\end{gathered}
$$

\footnotetext{
${ }^{4}$ Moran test results are available from the authors upon request.
} 
In comparing the forecasting power of competing models, it is important to take into account the scale heterogeneity in the unemployment rates of each province. It is thus convenient to consider also the MAPE, which considers forecasting errors on a percentage scale, and is given by the following:

$$
M A P E=\frac{1}{n} \sum_{i=1}^{n} \frac{\left|\hat{U}_{i}-U_{i}\right|}{U_{i}} \times 100 .
$$

Each model is tested on out-of-sample data for the years 2007 and 2008. Within this framework, the discussion on the advantages and disadvantages of ex-ante and ex-post predictions seems unnecessary, since contemporaneous spatial lags are obtained in the first stage, as described above. Angulo and Trívez (2010) avoid this debate as well, using a dynamic panel data model without explanatory variables to forecast employment levels in Spanish provinces.

On the basis of the above statistical indicators, we aim to generate inferential evidence on the relative forecasting performance of the two competing econometric models. Therefore, following Patuelli et al. (2008), we use a nonparametric test to assess if two models are equally accurate: the sign test (ST, Lehmann 1998). The sign test does not rely on the usual assumptions necessary for most comparison tests (such as the DieboldMariano test or the Wilcoxon test), as it does not require normal distribution or symmetry between the two vectors compared. More simply, the sign test is based on the comparison of the forecasting errors. If the methods tested present a similar forecasting performance, the number of SF (Model 2) forecasts which show a greater error than the one of SVAR (Model 1) may be expected to be 50 per cent of the total number of forecasts obtained. Consequently, Model 1 will be considered superior to Model 2 if Model 2 has higher forecasting errors in more than 50 per cent of the cases. Clearly, the test does not provide insights on the error distribution, but only on comparative forecasting, pairwise. In practice, what is being tested is the hypothesis of equality in the medians. The test statistic $S$ is computed as:

$$
S=\left(C-\frac{p}{2}\right) / \frac{\sqrt{p}}{2},
$$

where $C$ is the number of times that Model 2 shows a higher error than Model 1's, and $p$ is the number of forecasts carried out. The $S$ statistic follows a normal distribution $N(0,1)$. When not standardized, $\mathrm{C}$ follows a binomial distribution $B(p, 0.5)$. Confidence intervals for $S(C)$ are obtained in the standard way from the normal and binomial tests, respectively.

\subsection{Results for Switzerland}

Table 1 summarizes the statistical performance of SVAR and SF for the Swiss case, by means of MSE, MAE and MAPE, as we compute the cross-sectional average error for each of the 24 forecasting periods. Values in bold indicate the model with the lowest value. 
Table 1 - Summary statistics of MSE, MAE and MAPE for Switzerland

\begin{tabular}{|c|c|c|c|c|c|c|c|c|c|c|c|c|c|c|}
\hline \multirow{5}{*}{ } & & & I & II & III & IV & $\bar{V}$ & VI & VII & VIII & IX & $X$ & XI & XII \\
\hline & MSE & SVAR & 0.024 & 0.018 & 0.015 & 0.011 & 0.011 & 0.014 & 0.005 & 0.008 & 0.011 & 0.009 & 0.008 & 0.011 \\
\hline & & SF & 0.015 & 0.019 & 0.033 & 0.024 & 0.014 & 0.022 & 0.008 & 0.007 & 0.010 & 0.024 & 0.015 & 0.032 \\
\hline & & SF & 0.092 & 0.108 & 0.119 & 0.106 & 0.107 & 0.105 & 0.067 & 0.067 & 0.079 & 0.113 & 0.090 & 0.089 \\
\hline & MAPE & SVAR & 4.978 & 4.423 & 4.608 & 3.383 & 4.780 & 4.098 & 3.607 & 4.536 & 4.452 & 3.862 & 3.938 & 3.589 \\
\hline \multirow{5}{*}{$\stackrel{\infty}{8}$} & & & I & II & III & IV & $\mathrm{V}$ & VI & VII & VIII & IX & $X$ & XI & XII \\
\hline & MSE & SVAR & 0.016 & 0.014 & 0.003 & 0.012 & 0.011 & 0.006 & 0.006 & 0.005 & 0.007 & 0.016 & 0.007 & 0.041 \\
\hline & & SF & 0.012 & 0.021 & 0.015 & 0.017 & 0.013 & 0.012 & 0.008 & 0.006 & 0.007 & 0.025 & 0.015 & 0.086 \\
\hline & MAE & SVAR & 0.091 & 0.085 & 0.049 & 0.074 & 0.085 & 0.058 & 0.065 & 0.056 & 0.067 & 0.091 & 0.066 & 0.156 \\
\hline & & SF & 0.087 & 0.091 & 0.063 & 0.089 & 0.095 & 0.072 & 0.074 & 0.066 & 0.066 & 0.111 & 0.083 & 0.198 \\
\hline
\end{tabular}


From the analysis of Table 1, the SVAR model appears to show better forecasting performance than the SF model, although the difference between the two models is considerably reduced when the MAPE is considered. In any case, the numerical distance between the two models is rather small. A closer look at the heterogeneity of these forecasting errors is given by the inspection of the error distributions. Figure 2 collects the average MSE, MAE and MAPE for each estimation period and their confidence intervals. In all cases, the SF approach presents a high level of variability in comparison to the SVAR. In addition, Figure A.1 in the Appendix provides histograms for the aggregate distributions of the forecasting error indicators.

As a final analysis on the error indicators, the sign test is performed, depending on the forecasting errors used for comparison, along three dimensions. First, all forecasting errors are pooled (for all cross-sectional units and all forecasting periods). Then, the average forecasting errors by canton is analysed. Finally, the average forecasting errors per period are compared.

The pooled sign tests do no reject the hypothesis of equivalence in median between the SAR and SF model forecasts. The same conclusion is reached when the test is computed averaging the errors by forecasting period, but the results point out a statistically better performance of the SVAR model when the average forecasting errors by region are analysed.

Since both methods introduce explicitly account for spatial autocorrelation, we may expect that, if spatial patterning in the data is well identified, forecasting errors should not present spatial autocorrelation. To test this hypothesis, MI is computed, for each forecasting period, on the prediction errors of both methods.

Figure 3 summarizes our findings for the Swiss case. For the majority of forecasting periods there is no significant spatial autocorrelation, for both SVAR and SF models. However, SVAR seems to produce spatially autocorrelated forecasting errors in a smaller number of cases. This finding, joined with the evidence above on error indicators, is not surprising, since the time dimension is much larger than spatial dimension $(T \gg n)$ in the Swiss data set, therefore clearly advantaging a time-seriesrelated method like the SVAR. 



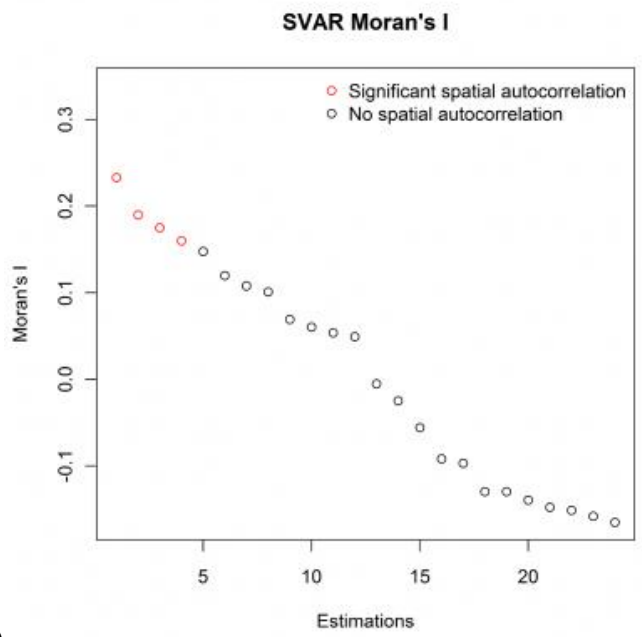

(a)

Figure 3 - MI of SVAR (a) and SF (b) forecasting errors for Switzerland, sorted in decreasing order

\subsection{Results for Spain}

Table 2 reports summary statistics for the MSE, MAE and MAPE over the eight forecasting periods (quarters) used for Spain. Our findings for Spain differ from the ones for Switzerland in that the SF model appears to have gained in competitiveness from the different data structure (the unbalance between $n$ and $T$ is now of a lesser extent). In particular, the SVAR model appears to be more competitive with regard to MSE and MAE (when the error is not standardized by the level of the unemployment rates), while the SF model minimizes percentage error (MAPE), winning six of out eight comparisons.

Once again, we can inspect the heterogeneity of forecasting errors, through the plots given in Figure 4. Differently from the Swiss case, it is now the SVAR model that presents a higher heterogeneity in forecasting errors. The increase in the cross-sectional dimension of the data set (from the 26 Swiss cantons to the 47 Spanish provinces) may be one reason for this finding, as spatial contiguity relationships become more meaningful in a richly disaggregated dataframe. Also noteworthy is the generalized increase in forecasting errors over time and in particular at the last two quarters, coinciding with the 2008 financial crisis, which had a strong labour market impact on the Spanish labour market. Figure A. 2 in the Appendix provides further evidence on the pooled statistical distribution of the forecasting errors obtained for Spain. 
Table 2 - Summary statistics of MSE, MAE and MAPE for Spain

\begin{tabular}{|c|c|c|c|c|c|c|}
\hline \multirow{7}{*}{ ঠ্ণ } & & & I & II & III & IV \\
\hline & MSE & SVAR & 1.883 & 1.344 & 1.097 & 1.244 \\
\hline & & SF & 1.630 & 1.341 & 1.279 & 1.850 \\
\hline & MAE & SVAR & 1.035 & 0.801 & 0.848 & 0.887 \\
\hline & & SF & 1.018 & 0.904 & 0.903 & 1.109 \\
\hline & MAPE & SVAR & 0.136 & 0.111 & 0.128 & 0.115 \\
\hline & & SF & 0.129 & 0.118 & 0.121 & 0.143 \\
\hline \multirow{7}{*}{ 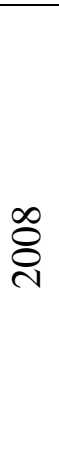 } & & & I & II & III & IV \\
\hline & MSE & SVAR & 1.779 & 2.981 & 2.459 & 6.002 \\
\hline & & SF & 1.942 & 2.654 & 2.479 & 5.035 \\
\hline & MAE & SVAR & 0.942 & 1.318 & 1.221 & 2.010 \\
\hline & & SF & 0.966 & 1.258 & 1.292 & 1.907 \\
\hline & MAPE & SVAR & 0.125 & 0.163 & 0.135 & 0.196 \\
\hline & & SF & 0.122 & 0.154 & 0.134 & 0.188 \\
\hline
\end{tabular}

We compute the sign test to assess whether our two methods can be considered as equally accurate when forecasting Spanish unemployment rates. The main difference with the Swiss case lies in the temporal and cross-sectional dimension, since we now deal with moderate values of both $n$ and $T$. We calculate the test using all forecasting errors (pooled test), the average forecasting errors by region, and the average forecasting errors by quarter. In all cases, the tests are not significant, suggesting an overall equivalence between the SVAR and the SF models.

Finally, Figure 5 plots the MI statistic computed for each (of the eight) forecasting periods. As for the case of Switzerland, both competing methods produce spatially uncorrelated forecasting errors in most cases, but the SVAR model appears to account better for the true spatial correlation in the dependent variable. In any case, the levels of spatial autocorrelation of forecasting errors, when significant, are very low. 

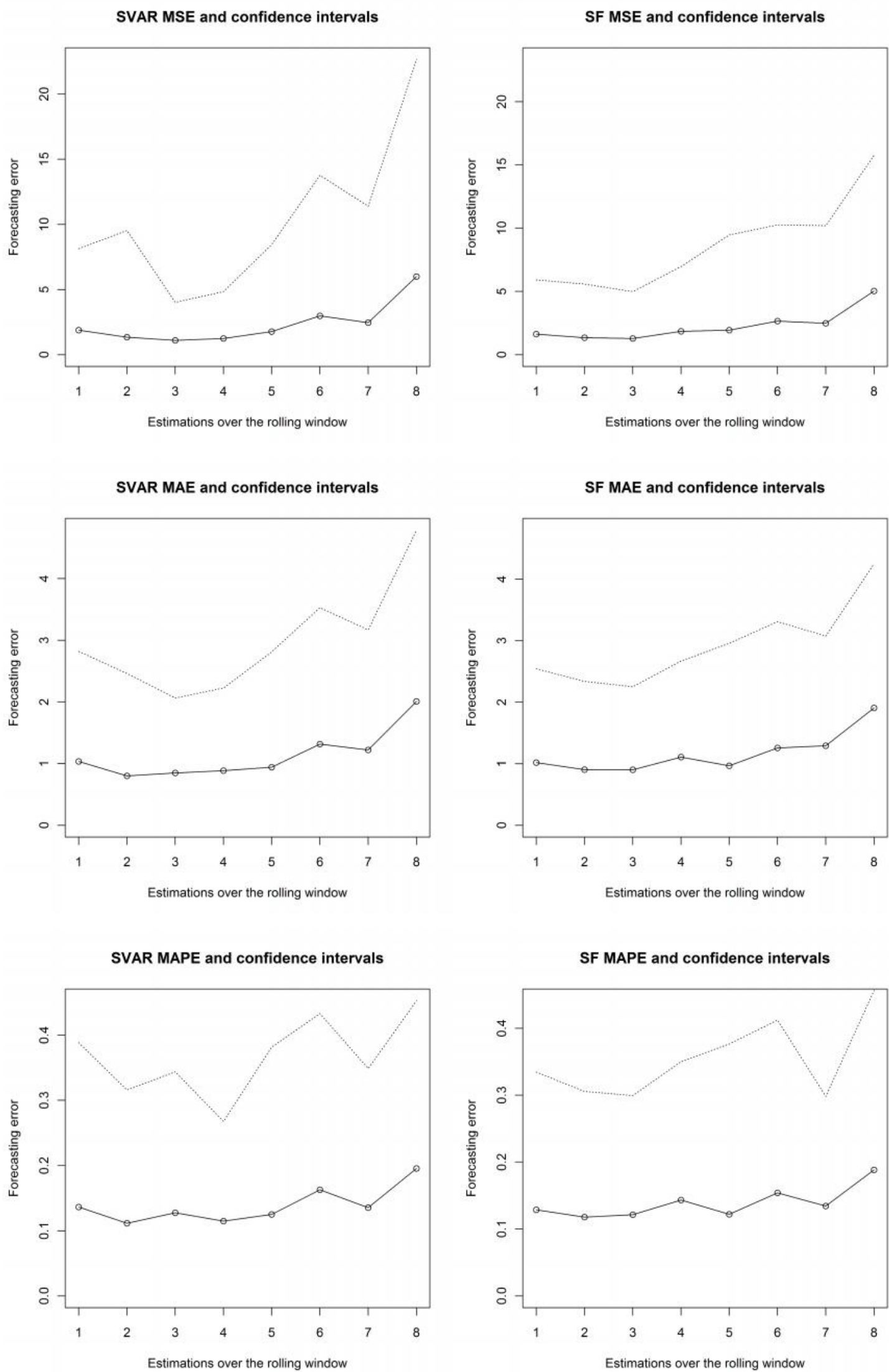

Figure 4 - MSE, MAE, MAPE and confidence intervals for SVAR (left) and SF (right) models for Spain 


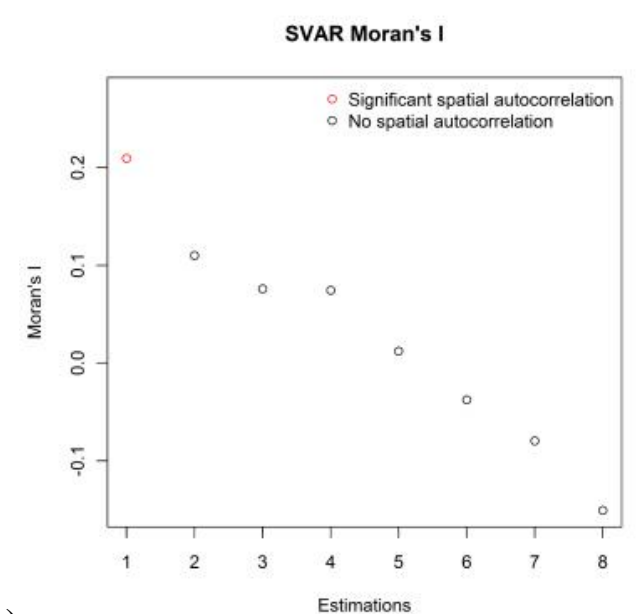

(a)

Figure 5 - MI of SVAR (a) and SF (b) forecasting errors for Spain, sorted in decreasing order

\section{Rejoinder and Conclusions}

The aim of this paper was to analyse the short-run forecasting performance of two competing spatial models: a spatial vector-autoregressive model (SVAR) and a dynamic panel data model employing spatial filtering (SF). We developed a sensitivity analysis in order to test how different number and size of the spatial units (actually administrative areas) and varying extent of the temporal dimension influence the relative forecasting performance of these methods.

Our empirical application used regional unemployment rates at the NUTS-3 level of geographical aggregation for two countries: Spain and Switzerland. Switzerland has a low number of NUTS-3 regions (26), which are also much smaller in areal extension than their (47) Spanish equivalents. Moreover, although the data in both data sets are collected for a similar number of years, Swiss unemployment data have a month frequency, while Spanish data are quarterly, resulting in rather different data structures: for Switzerland, $T \gg n$, while for Spain this data unbalance is smaller, as there is some level of convergence between $T$ and $n$.

We carried out one-period-ahead ex post forecasts for the years 2007 and 2008, using a fixed rolling window to estimate both models. Our results were evaluated by means of statistical indicators (MSE, MAE and MAPE), as well as a forecast equivalence test (sign test).

From an empirical viewpoint, the aforementioned differences in data structure between the Swiss and Spanish data sets appears to be a discriminating factor in terms of forecasting accuracy. The SVAR model seems slightly preferable on the SF model when the temporal dimension is much greater than the spatial dimension and the spatial units have smaller size and greater degree of variability (i.e., the Swiss data). Although not on a consistent basis, sign test results support this finding.

When moderate cross-sectional and time dimensions were used (i.e., the Spanish data), we did not find stable significance differences between the two competing methods. The SVAR models appear to minimize errors on the scale of the unemployment rates (MSE and MAE), while the SF model is preferable when percentage error is considered (MAPE). This finding is justified by methodological aspects, as the SF model computes 
a geographical approximation of both the autoregressive coefficients and of the fixed/random effects usually employed in dynamic panel data models. As such, it may be less efficient in estimating outliers (e.g., change in high unemployment areas), while it may be expected to provide smoother findings on the spatial patterning of coefficients.

Finally, we investigated whether the spatial patterns of the data were well-identified in both methods, testing the existence of spatial autocorrelation in the forecasting errors. The SVAR model shows a lesser number of spatially autocorrelated errors for both the Swiss and the Spanish data sets, although most estimations produced uncorrelated errors for both methods.

In summary, the SVAR models showed somehow superior performance when the time dimension was clearly dominant on the cross-sectional dimension, consistently with the underlying time-series framework of VAR models. Moving instead to moderate crosssectional and temporal dimensions, no clear difference can be drawn between the SVAR and SF estimation frameworks. It was not possible, within this study, to test a data structure opposite to the one of Switzerland (e.g., German NUTS-3 unemployment data, which were available to the authors, and for which $n \gg T$ ), as VAR models cannot be estimated in such case.

Further research should complement this preliminary empirical investigation. From a methodological viewpoint, it is desirable to expand our study to include more (spatial and non-spatial) econometric models (e.g., spatial panel data models, a la Elhorst). From an empirical viewpoint, out findings should be verified through a simulation study, which could allow for a greater variability in cross-sectional and temporal size, to provide a more complete 'map' of the comparative performance of spatial models in forecasting. Finally, expanding the forecasting horizon would allow us to test whether the relative forecasting relationships found in this paper are preserved for longer-periods forecasts.

\section{References:}

Angulo, A. and Trívez, F. J. (2010). The Impact of Spatial Elements on the Forecasting of Spanish Labour Series. Journal of Geographical Systems, 12(2), 155-74.

Baltagi, B. H. and Li, D. (2004). Prediction in Panel Data Model with Spatial Correlation. In L. Anselin, R. J. G. M. Florax and S. J. Rey (Eds.), Advances in Spatial Econometrics: Methodology, Tools and Application (pp. 283-95). Berlin Heidelberg: Springer-Verlag.

Baltagi, B. H. and Li, D. (2006). Prediction in the Panel Data Model with Spatial Correlation: the Case of Liquor. Spatial Economic Analysis, 1(2), 175-85.

Beenstock, M. and Felsenstein, D. (2007). Spatial Vector Autoregressions. Spatial Economic Analysis, 2(2), 167-96.

Blanchard, O. and Jimeno, J. F. (1995). Structural Unemployment: Spain versus Portugal. The American Economic Review, 85(2), 212-18.

Di Giacinto, V. (2003). Differential Regional Effects of Monetary Policy: A Geographical SVAR Approach. International Regional Science Review, 26(3), 313-41. 
Elhorst, J. P. (1995). Unemployment Disparities between Regions in the European Union. In H. W. Armstrong and R. W. Vickerman (Eds.), Convergence and Divergence among European Unions (pp. 209-21). London: Pion.

Ertur, C. and Le Gallo, J. (2003). An Exploratory Spatial Data Analysis of European Regional Disparities, 1980-1995. In B. Fingleton (Ed.), European Regional Growth (pp. 55-98). Berlin Heidelberg New York: Springer-Verlag.

Fingleton, B. (2009). Prediction Using Panel Data Regression with Spatial Random Effects. International Regional Science Review, 32(2), 195-220.

Fotheringham, A. S., Brunsdon, C. and Charlton, M. (2002). Geographically Weighted Regression: The Analysis of Spatially Varying Relationships. Chicester: John Wiley \& Sons Ltd.

Giacomini, R. and Granger, C. W. J. (2004). Aggregation of Space-Time Processes. Journal of Econometrics, 118(1-2), 7-26.

Griffith, D. A. (2000). A Linear Regression Solution to the Spatial Autocorrelation Problem. Journal of Geographical Systems, 2(2), 141-56.

Griffith, D. A. (2003). Spatial Autocorrelation and Spatial Filtering: Gaining Understanding through Theory and Scientific Visualization. Berlin New York: Springer.

Griffith, D. A. (2008). Spatial-Filtering-Based Contributions to a Critique of Geographically Weighted Regression (GWR). Environment and Planning A, 40(11), 2751-69.

Hernández-Murillo, R. and Owyang, M. T. (2006). The Information Content of Regional Employment Data for Forecasting Aggregate Conditions. Economics Letters, 90(3), 335-39.

Jimeno, J. F. and Bentolila, S. (1998). Regional Unemployment Persistence (Spain, 1976-1994). Labour Economics, 5(1), 25-51.

Kholodilin, K. A., Siliverstovs, B. and Kooths, S. (2008). A Dynamic Panel Data Approach to the Forecasting of the GDP of German Länder. Spatial Economic Analysis, 3(2), 195-207.

Kuethe, T. H. and Pede, V. O. (2011). Regional Housing Price Cycles: A Spatiotemporal Analysis Using US State-level Data. Regional Studies, 45(5), 563-74.

Lehmann, E. L. (1998). Nonparametrics: Statistical Methods Based on Ranks (rev. ed.). Upper Saddle River: Prentice Hall.

Longhi, S. and Nijkamp, P. (2007). Forecasting Regional Market Developments under Spatial Autocorrelation. International Regional Science Review, 30(2), 100-51.

López-Bazo, E., del Barrio, T. and Artis, M. (2002). The Regional Distribution of Spanish Unemployment: A Spatial Analysis. Papers in Regional Science, 81(3), 36589.

Molho, I. (1995). Spatial Autocorrelation in British Unemployment. Journal of Regional Science, 35(4), 641-58.

Moran, P. (1948). The Interpretation of Statistical Maps. Journal of the Royal Statistical Society B, 10, 243-51. 
Overman, H. G. and Puga, D. (2002). Unemployment Clusters across Europe's Regions and Countries. Economic Policy, 17(34), 115-48.

Pan, Z. and LeSage, J. P. (1995). Using Spatial Contiguity as Prior Information in Vector Autoregressive Models. Economics Letters, 47(2), 137-42.

Patacchini, E. and Zenou, Y. (2007). Spatial dependence in local unemployment rates. Journal of Economic Geography, 7(1), 169-191.

Patuelli, R. (2007). Regional Labour Markets in Germany: Statistical Analysis of Spatio-Temporal Disparities and Network Structures. Unpublished Ph.D. Thesis, Department of Spatial Economics, VU University Amsterdam, Amsterdam.

Patuelli, R., Longhi, S., Reggiani, A. and Nijkamp, P. (2008). Forecasting Regional Employment in Germany by means of Neural Networks and Genetic Algorithms. Environment \& Planning B, 35(4), 701-22.

Patuelli, R., Schanne, N., Griffith, D. A. and Nijkamp, P. (2012). Persistence of Regional Unemployment: Application of a Spatial Filtering Approach to Local Labour Markets in Germany. Journal of Regional Science, forthcoming.

Pesaran, M. H., Schuermann, T. and Weiner, S. M. (2004). Modeling Regional Interdependencies Using a Global Error-Correcting Macroeconometric Model. Journal of Business and Economic Statistics, 22(2), 129-62.

Schanne, N., Wapler, R. and Weyh, A. (2010). Regional Unemployment Forecasts with Spatial Interdependencies. International Journal of Forecasting, 26(4), 908-26.

Sims, C. A. (1980). Macroeconomics and Reality. Econometrica, 48(1), 1-48.

Taylor, J. and Bradley, S. (1997). Unemployment in Europe: A Comparative Analysis of Regional Disparities in Germany, Italy and the UK. Kyklos, 50(2), 221-45.

Tiefelsdorf, M., Griffith, D. A. and Boots, B. N. (1999). A Variance Stabilizing Coding Scheme for Spatial Link Matrices. Environment and Planning A, 31, 165-80. 


\section{Appendix}
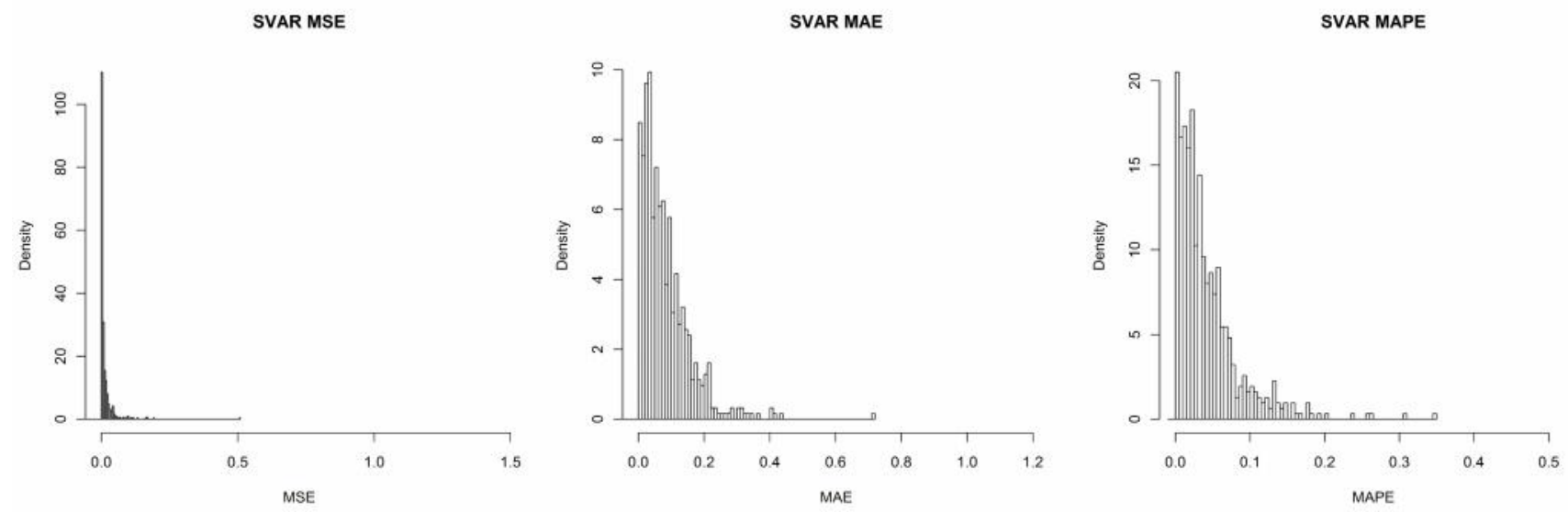

SF MSE
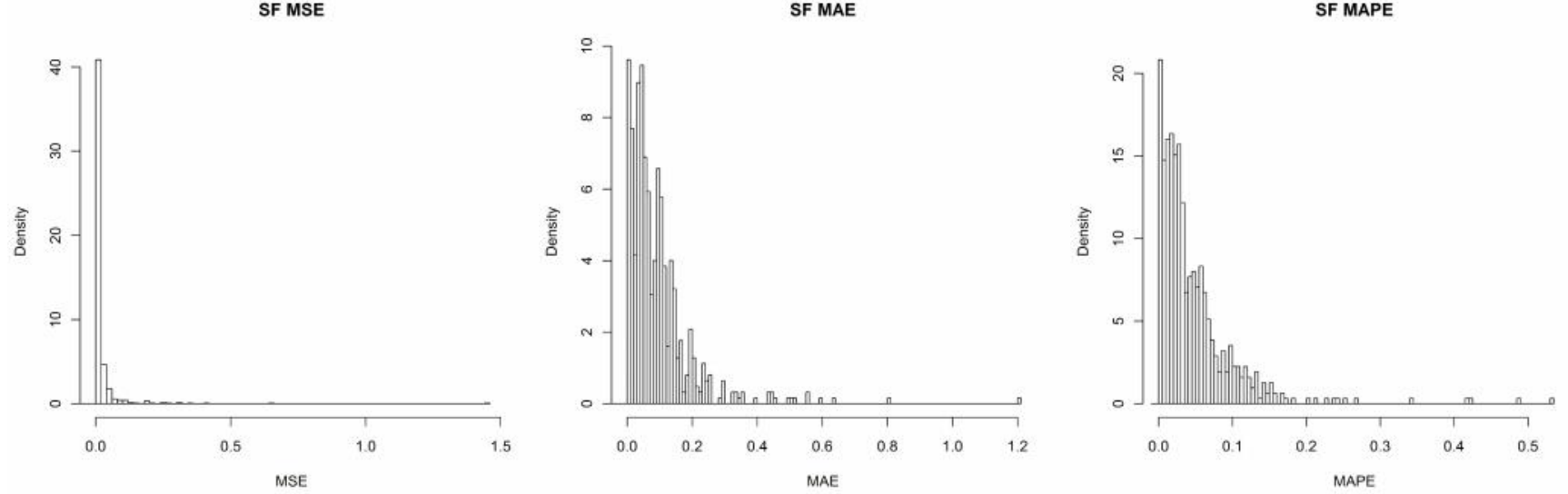

Figure A.1 - Histograms of MSE, MAE and MAPE of SVAR (above) and SF (below) models for Switzerland 
SVAR MSE

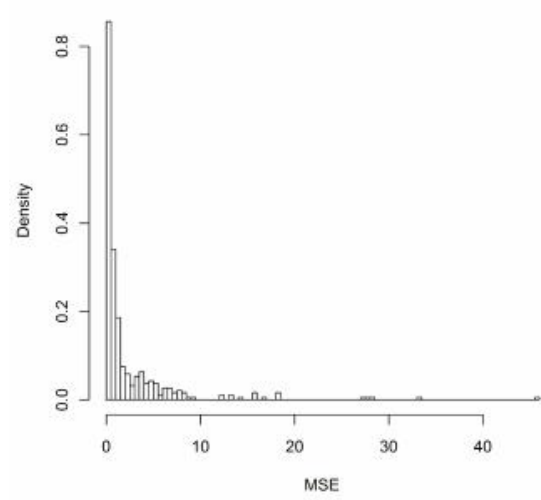

SF MSE

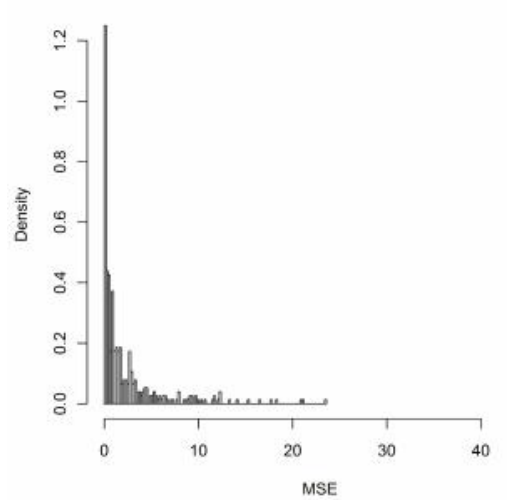

SVAR MAE

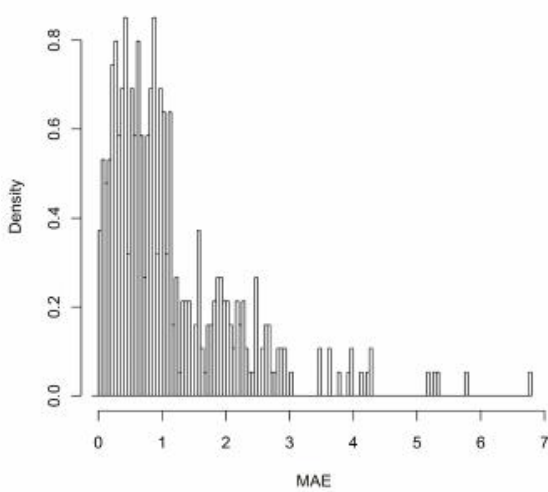

SF MAE

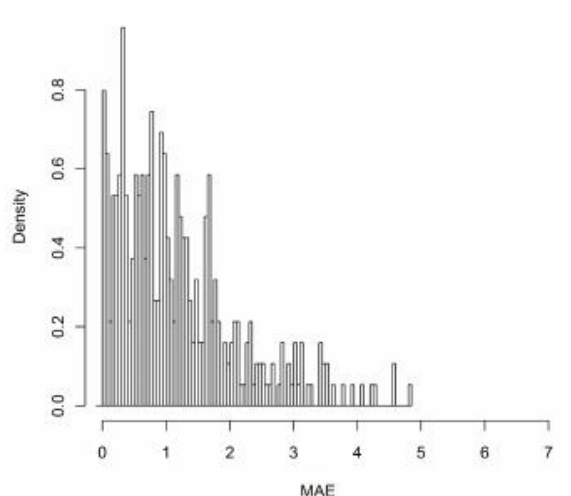

SVAR MAPE

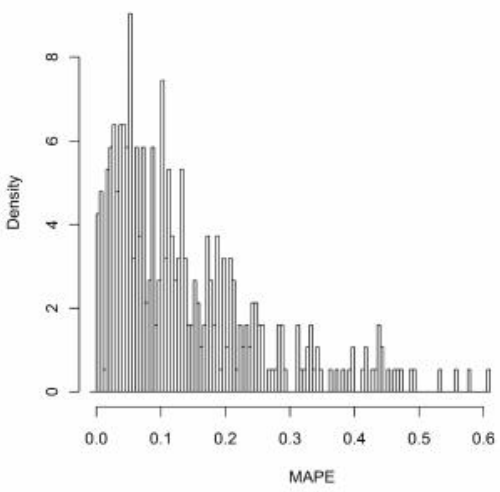

SF MAPE

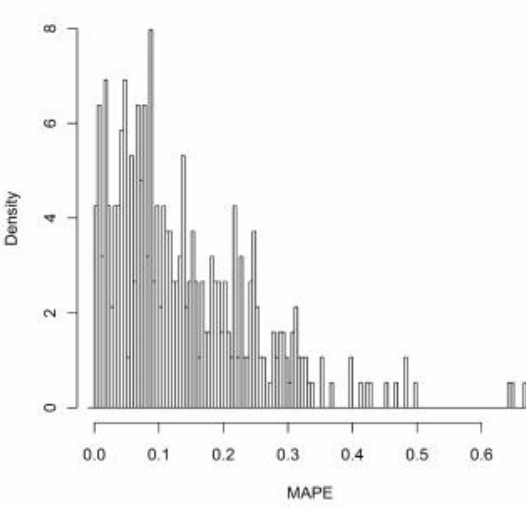

Figure A.2 - Histograms of MSE, MAE and MAPE of SVAR (above) and SF (below) models for Spain 


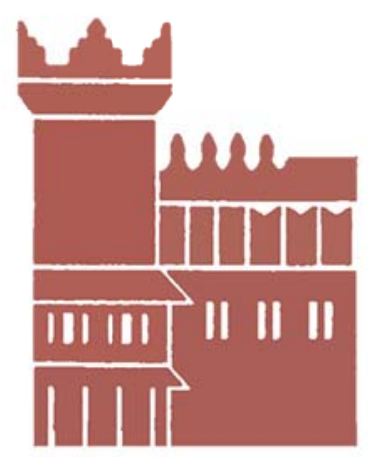

Alma Mater Studiorum - Università di Bologna DEPARTMENT OF ECONOMICS

Strada Maggiore 45

40125 Bologna - Italy

Tel. +39051 2092604

Fax +390512092664

http://www.dse.unibo.it 